\title{
Sensitive Variable Analysis for Comprehensive optimization of an SWATH-USV
}

\author{
Wang Baojiang, Tang Xiongfeng, Yang Songlin, Qiu Menglong
}

\begin{abstract}
In this paper, aiming at the comprehensive optimization of SWATH, we establish a optimization mathematical model of SWATH. And use genetic algorithm to carry out the comprehensive optimization calculation. To solve the problem of premature convergence of genetic algorithm, we adopt parallel strategy to improve the efficiency of the algorism on three design variables: Loa, speed item. In order to improve the parallel efficiency of the algorithm, we have carried out sensitivity analysis propeller diameter, and obtained the analysis results. The research results can provide a reference for the multi-objective, multi-variable and multi-constrained comprehensive optimization of the performance optimization of SWATH.
\end{abstract}

Index Terms-Swath-USV; Comprehensive optimization; Genetic algorithm; Parallel strategy; Sensitive variables;

\section{INTRODUCTION}

Small Waterline Catamaran (SWATH-USV) was first built in 1973 and developed rapidly in the 1980s. As a high performance ship, small water plane catamaran has the advantages of good wave resistance, high propulsion efficiency, large hull deck area and good transverse stability. For Small Waterline Catamaran, it is of great significance to obtain the optimal ship type and improve the performance by scientific and effective calculation. In the process of ship form optimization research, Ma Weijia [1], hua-wei sun, yong-jie pang, Guo Zhiqun, Yang Heng transformed the boat optimization problem under given constraints into a constrained mathematical optimization problem for finding the functional extremum. Through nonlinear programming optimization theory, The objective function constrained by the given main parameter type. And they find the optimal ship form. Yang zhuoyi [2], pang yongjie, wang jian and song lei studied the experimental design, response surface model technolometers of the hull is minimized conditionally to solve the optigy and multi-objective optimization algorithm for the submarine's boat type optimization. In terms of optimization algorithm, genetic algorithm, as a random search and optimization method based on biological natural selection and genetic mechanism, has high efficiency in solving complex problems. Chen kaifeng [3] discussed various implementation methods using genetic algorithm and compared their advantages and disadvantages, demonstrating the effectiveness of genetic algorithm in solving multi-objective optimization problems. Goldberg[4] proposed the idea of designing parallel structure of genetic algorithm based on object. Aiming at the problem of premature convergence of genetic algorithm, zhou yuanhui [5], lu yuchang and shi chunyi proposed the idea of combining multi-population

Wang Baojiang, Tang Xiongfeng, Yang Songlin, Qiu Menglong, School of Naval Architecture \& Ocean Engineering , Jiangsu University of Science and Technology, Zhenjiang, Jiangsu, China parallel evolution and adaptive adjustment control parameters to alleviate the problem of premature convergence. It improves the efficiency of the non-inferior hierarchical multi-objective genetic algorithm and makes up for the disadvantages of the genetic algorithm, such as poor local search ability and precocity.

For the problem of multi-objective comprehensive optimization in SWATH-USV, this paper selects corresponding design variables to establish a comprehensive optimization mathematical model, selects genetic algorithm as the optimization algorithm, and seeks for sensitive variables in Loa $\mathrm{L}$, design speed $\mathrm{V}$ and propeller diameter $D p$, so as to improve the parallel efficiency of the algorithm.

\section{OPTIMIZATION MODEL OF SWATH-USV}

\section{A. Rapidity}

According to the theory and experience, this paper synthetically considers the selection of design variables: the length of the submerged body $L_{h}$, the diameter of the submerged body $D_{1}$, The length of the pillar $L_{s}$, Maximum strut width $t_{s}$, the Loa $L$, the width of the $\operatorname{ship} B$, the $\operatorname{draft} T$, the longitudinal position of the buoyancy center $L_{c p}$, the diameter of the propeller $D_{P}$, the disk-to-surface ratio $A_{e o}$, the pitch ratio $D_{P}$, the speed of the propeller $N$, and the designed speed $V_{S}$. Represented by a vector:

$$
X_{s p}=\left\{L_{h}, D_{1}, L_{s}, t_{s}, L, B, T, L_{c b}, D_{p}, A_{e o}, P_{d p}, N, V_{s}\right\}
$$

The objective function takes the speediness criterion factor similar to the naval coefficient form as the speediness optimization objective function:

$$
\begin{gathered}
f_{1}(x)=C_{s p}=\frac{V_{s}^{3} \Delta^{\frac{2}{3}}}{p_{E}}= \\
\frac{V_{S}^{2} \Delta\left(\eta_{0} \eta_{H} \eta_{R} \eta_{S}\right)}{R_{t} V_{s} * 0.5144 * 1.35962 * 10^{-3}}
\end{gathered}
$$

In the(2-fformula : Effective power $P_{E}$ (unit: horsepower); $\Delta$ Drainage (unit: ton); $V_{s}$ Design voyage (unit: knot); $R_{t}$ Hull resistance (unit: N); $\eta_{0}$ Open water efficiency of propeller; $\eta_{H}$ Hull efficiency; $\eta_{R}$ Relative rotation efficiency; $\eta_{s}$ Shafting efficiency. 


\section{B. Seakeeping}

In this paper, the rolling natural period is selected as the objective function for the study of the mathematical model of seakeeping. Selection of design variables: Loa $L$, ship width $\mathrm{B}$, draft $T$, square coefficient $C_{B}$, waterline coefficient $C_{w}$, demi hull spacing $C_{0}$, center of gravity height of catamaran $Z_{g}$. Represented by vector $X_{s p}$, that is:

$$
X_{S P}=\left\{L, B, T, C_{b}, C_{w}, c 0, Z_{g},\right\}
$$

For catamaran, its stability is good, but its rolling cycle is short, especially at high speed. In this paper, $f_{2}(x)^{\text {-the }}$ natural period of a ship is chosen as the objective function of seakeeping, and its expression is as follows:

$$
f_{2}(x)=T_{\phi}=\frac{2 \pi}{w_{\phi}}=2 \pi \sqrt{\frac{I_{x x}^{\prime}}{\Delta h}}(s)
$$

In the formula, the natural rolling period is $\omega_{\phi}=\sqrt{(\Delta h) / I_{X X}^{\prime}}\left(s^{-1}\right)$, the sum of inertia moment and additional inertia moment of the hull itself is $I_{x x}^{\prime}$, displacement is $\Delta$, the initial stability of the ship is $h$, the inertia moment of the hull itself is $I_{x x}$, and the additional inertia moment is $J_{x x}$.

\section{Maneuverability}

In this paper, heading stability is taken as the research object of maneuverability to select design variables. Considering comprehensively, the selected design variables are Loa $L$, ship width $B$, draft $T$, square coefficient $C_{b}$ and water line length $L_{w}$. Representation by a vector:

$$
X_{S P}=\left\{L, B, T, C_{b}, L_{w}\right\}
$$

We selected linear stability as the objective function of maneuverability of Small waterplane catamaran, which is expressed by the linear stability criterion coefficient $C$, Linear motion stability is determined by the changing characteristics of state variables $v$, and $r$. The stability of the system is directly related to the four acceleration hydrodynamic derivatives and the four velocity hydrodynamic derivatives $Y_{v}^{\prime}, Y_{r}^{\prime}, N_{v}^{\prime}, N_{r}^{\prime}$, which can be expressed by the linear stability criterion coefficients of ships C :

$$
f_{3}=c=Y_{v}^{\prime} N_{r}^{\prime}-N_{v}^{\prime}\left(Y_{r}^{\prime}-m^{\prime}\right)(2-3)
$$

\section{Constraints}

\section{(1) Equality constraints}

(1) Floating constraints in static water, i.e. the drainage obtained by USV optimization is consistent with the design drainage:

$$
\Delta=\mathrm{LBHC}_{b} \rho
$$

(2) Thrust resistance balance constraint, that is, the effective thrust of the propeller is equal to the navigation resistance of the hull:

$$
N_{p} K_{T} \rho N^{2} D_{P}^{4}(1-t)=R_{t}
$$

(3) Torque balance constraint, i.e. the torque supplied by the main engine to the propeller is equal to the hydrodynamic torque endured by the propeller

$$
\frac{\eta_{R} \eta_{s} P_{s}}{2 \pi N}=K_{Q} \rho N^{2} D_{p}^{5}
$$

\section{(2) Inequality constraint}

The propeller needs to satisfy the requirement of cavitation, which is derived from Keller's formula:

$$
(1.3+0.3 Z) T_{e} /\left(\left(P_{0}-P_{V}\right) D_{P}^{2}\right)+K=\left(A_{E} / A_{0}\right)_{\min }
$$

Then, the inequality constraint condition is:

$$
(1.3+0.3 Z) T_{e} /\left(\left(P_{0}-P_{V}\right) D_{P}^{2}\right)+K-\left(A_{E} / A_{0}\right) \leq 0
$$

In the formula: the static pressure at the center of propeller shaft is $P_{0}$; the vaporization pressure of sea water at 15 degrees Celsius is $P_{V}$; the thrust of propeller is $T_{e}$; the blade number of propeller is $Z$; the constant is $K$, the fast boat is 0 , others, twin-propeller boats are 0.1 , and the single-propeller boat is 0.2 .

According to the ship code, Initial stability height $G M>0.7 m$

Rolingl cycle:

$$
T_{\varphi}=1.05 B / \sqrt{G M}<6.0 s
$$

\section{E. Optimize the total objective function}

The objective functions of the three subsystems of speediness, maneuverability and seakeeping of UAV are synthesized, and the general objective function is $f(x)$ constructed in the form of power exponential product as follows :

$$
f(x)=f_{1}(x)^{\alpha 1} \cdot f_{2}(x)^{\alpha 2} \cdot f_{3}(x)^{\alpha 3}
$$

In the formula: $\alpha_{1}, \alpha_{2}, \alpha_{3}$ are weights of rapidity, maneuverability and Seakeeping respectively. In the form of product sum $\alpha_{1} \times \alpha_{2} \times \alpha_{3}=1$, this paper needs to optimize the value of the total objective function [7] $f(x)$ as large as possible.

\section{ANALYSIS OF GENETIC AlgORITHMS}

\section{A. Introduction of Genetic Algorithms}

The basic idea of genetic algorithm is mainly derived from Darwin's evolutionism, which is an algorithm formed by simulating the biological inheritance and evolutionary mechanism in nature. Its principle is to produce better individuals according to the mechanism of eliminating the fittest. In the algorithm, according to the fitness value of the individual in the problem, the selected individuals are inherited to the next generation by certain rules, and the selected individuals are randomly manipulated by crossover and mutation, thus generating a new population solution [8-9].

The steps of standard genetic algorithm are as follows: 1) Random initialization of population. 2) Calculate the fitness of each individual in the population. 3) When the optimal solution is found or the maximum genetic algebra is reached, 
the algorithm is terminated, the loop is withdrawn and the optimal individual is output. 4) Otherwise, the roulette is selected according to the fitness value. 5) Cross and mutation operations. 6) Return to step 3. The flow chart of the algorithm is shown in Figure 3.1.

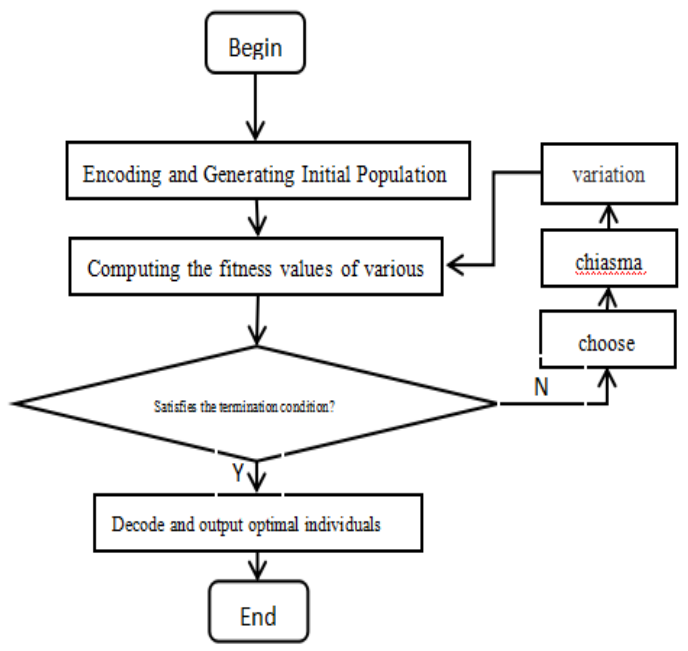

Figure 3.1 flow chart of genetic algorithm

\section{B. Parallel Strategy of Genetic Algorithms}

The genetic algorithm has good global search ability and can search all the solutions in the solution space quickly. But in practical application, genetic algorithm is prone to premature convergence. The premature problem is the problem of local convergence. In the process of optimization calculation, premature convergence and local optimum are not conducive to finding the optimal solution in the overall situation, and parallel strategy can alleviate such problems. Parallel strategy is to divide and parallel out multiple subspaces in the solution space of multiple variables, and then find the optimal solution sequentially in each subspace combination. If the parallel number is $\mathrm{P}-1$, the interval of each variable is divided into $\mathrm{P}$ (see Figure 3.2). For $\mathrm{N}$ design variables, the interval of optimization calculation has PN combinations. If the number of optimization calculation is $\mathrm{M}$ times, the number of calculation of the whole optimization system is $\mathrm{M}^{*} \mathrm{PN}$ times.

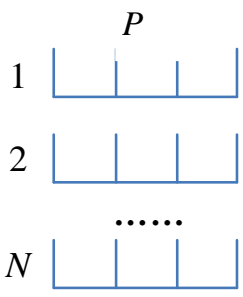

Figure 3.2 parallel thinking

\section{Introduction to optimization software}

The composition of the whole software includes the following Windows: the software enters the interface (FIG. 3.3), the main operation window (FIG. 3.4), the weight setting window (FIG. 3.5), the penalty factor setting window (FIG. 3.6), the upper and lower limit setting window of design variables (FIG. 3.7), and the calculation interface of each algorithm (FIG. 3.8).

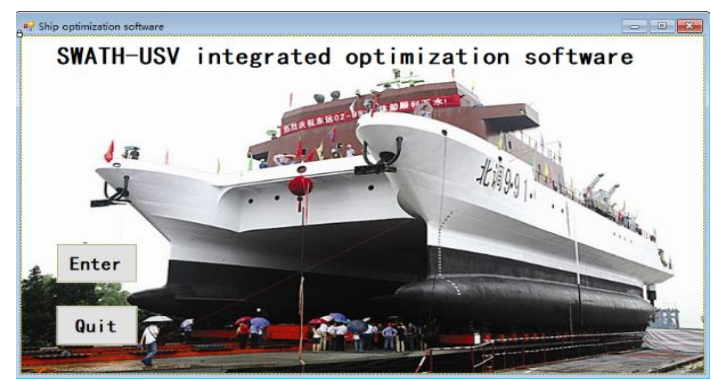

Figure 3.3 interface diagram of the integrated optimization program

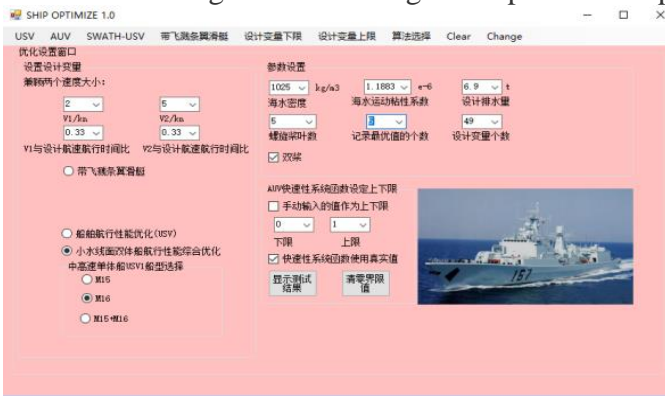

Figure 3.4 main interface of integrated optimization program operation

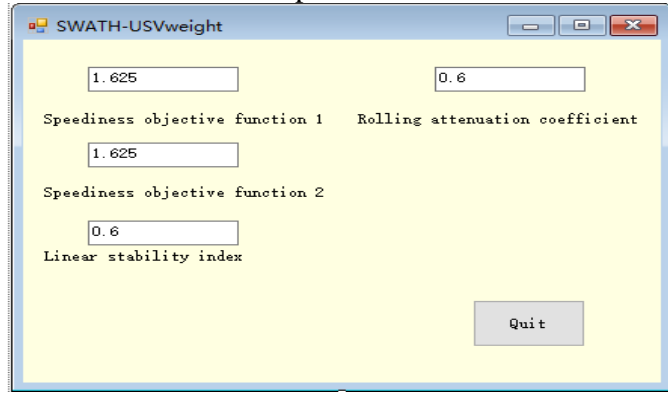

Figure 3.5 performance weight Settings

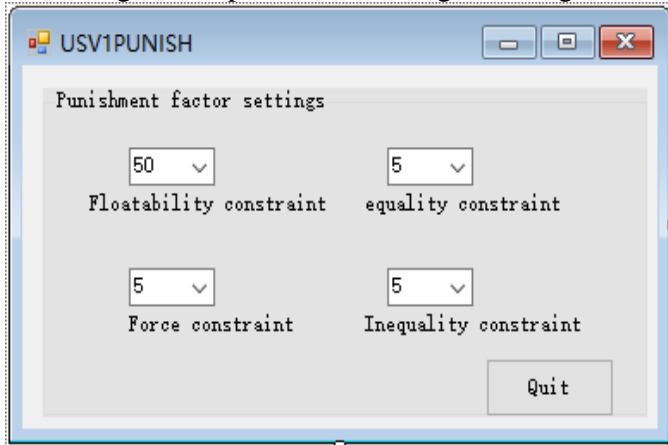

Figure 3.6 penalty factor setting window

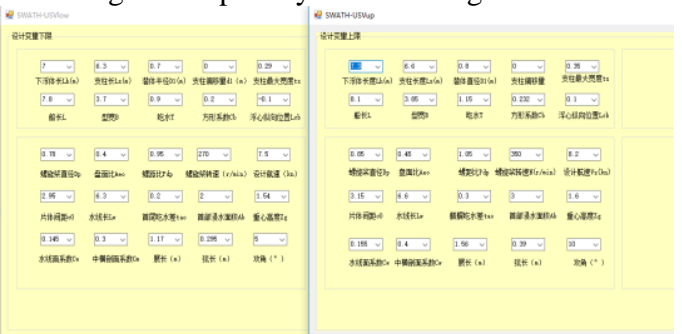

Figure 3.7 upper and lower limit setting Windows of design variables

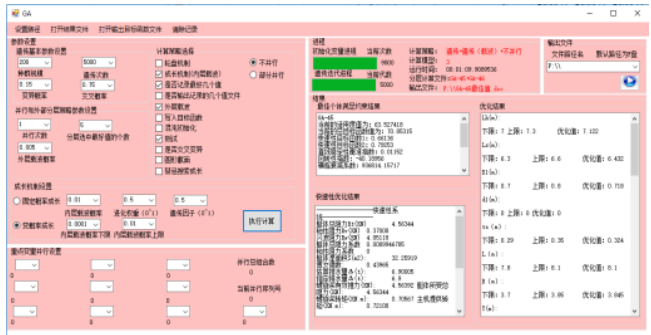

Figure 3.8 optimization calculation window of genetic algorithm 


\section{EXPLORE SENSITIVE VARIABLES}

By applying parallel strategy to genetic algorithm, the problem of local optimum can be solved. The key to parallel design is to find sensitive variables. For small waterplane catamarans, in the design variables selected in this paper. According to experience, Loa L, design speed Vs and propeller diameter $\mathrm{Dp}$ are three variables that are more sensitive to the objective function. The main work of this paper is to find the most sensitive variable to the objective function among these three variables, which will provide help for the parallel design of genetic algorithm.

The initial settings and optimum values of each variable are as follows.

\begin{tabular}{|c|c|c|c|}
\hline Variable & $\begin{array}{l}\text { Lower limit } \\
\text { of variables }\end{array}$ & $\begin{array}{l}\text { Upper limit } \\
\text { of variables }\end{array}$ & $\begin{array}{c}\text { Optimization } \\
\text { value }\end{array}$ \\
\hline Submergence length $\operatorname{Lh}(\mathrm{m})$ & 7 & 7.3 & 7.187 \\
\hline Prop length Ls(m) & 6.3 & 6.6 & 6.349 \\
\hline Submersible radius $\mathrm{Dl}(\mathrm{m})$ & 0.7 & 0.8 & 0.783 \\
\hline Pillar offset d1(m) & 0 & 0 & 0 \\
\hline Loa L（m） & 7.8 & 8.1 & 8.083 \\
\hline Type width B (m) & 3.7 & 3.85 & 3.715 \\
\hline Draught $\mathrm{T}(\mathrm{m})$ & 0.9 & 1.15 & 0.908 \\
\hline Block coefficient $\mathrm{Cb}(\mathrm{m})$ & 0.2 & 0.232 & 0.222 \\
\hline $\begin{array}{l}\text { longitudinal center on } \\
\text { buoyancy Lcb }\end{array}$ & -0.1 & 0.1 & 0.086 \\
\hline Propeller diameter dp & 0.84 & 0.85 & 0.847 \\
\hline Area ratio Aeo & 0.4 & 0.45 & 0.447 \\
\hline Pitch ratio Pdp & 1.1 & 1.15 & 1.126 \\
\hline Propeller speed N(r/min) & 270 & 350 & 279.22 \\
\hline Design speed Vs(kn) & 7.5 & 8.2 & 7.994 \\
\hline Segment spacing c0(m) & 2.95 & 3.15 & 3.085 \\
\hline Line length $\mathrm{Lw}(\mathrm{m})$ & 6.3 & 6.6 & 6.38 \\
\hline Submerged area of bow $\mathrm{Ab}$ & 2 & 3 & 2.204 \\
\hline $\begin{array}{l}\text { The center of gravity height } \\
\mathrm{Zg}(\mathrm{m})\end{array}$ & 1.54 & 1.6 & 1.583 \\
\hline Waterplane coefficient $\mathrm{Cw}$ & 0.145 & 0.155 & 0.152 \\
\hline
\end{tabular}

Table 4-1 upper and lower limits of initial design variables and optimization table

The process of finding sensitive variables is as follows: The interval of other variables is controlled unchanged. The Loa $\mathrm{L}$ is divided into the following seven intervals for optimization. The interval and the corresponding function value a are shown in Table 4-2. The trend of variation is shown in Figure 4-1 below.

\begin{tabular}{cccccccc}
\hline $\mathrm{L}$ & $7.8-7.8$ & $7.85-7.85$ & $7.9-7.9$ & $7.95-7.95$ & $8.0-8.0$ & $8.05-8.05$ & $8.1-8.1$ \\
$\mathrm{a}$ & 100.86 & 102.04 & 103.11 & 107.18 & 111.77 & 113.90 & 109.23 \\
\hline
\end{tabular}

Table 4-2 Loa sensitivity test table function value A

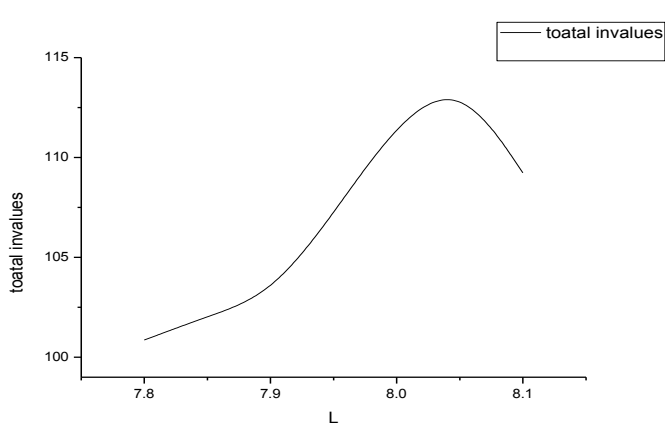

Figure 4-1 trend diagram of the total objective
As can be seen, when the value of variable Loa $L$ is in the interval [7.8-8.05]. The total objective function shows an increasing trend and reaches its maximum value at [8.05-8.05]. In the following interval [8.05-8.1], the value of the total objective function decreases. Therefore, when the Loa is within the interval $[7.8,8.1]$, it is the best choice to take the value of Loa around 8.05 to optimize the value of the total objective function.

The interval of other variables is controlled unchanged.Controlling other variable intervals unchanged, the design speed $\mathrm{Vs}$ is divided into the following seven intervals for optimization. The interval and the corresponding function value $b$ are shown in Table 4-3. The trend of variation is shown in Figure 4-2 below.

\begin{tabular}{cccccccc}
\hline $\mathrm{V}$ & $7.5-7.7$ & $7.61-7.61$ & $7.73-7.73$ & $7.85-7.85$ & $7.97-7.97$ & $8.09-8.09$ & $8.2-8.2$ \\
$\mathrm{~s}$ & & & 116.87 & 98.95 & 106.76 & 98.28 & 95.76 \\
$\mathrm{~b}$ & 123.07 & 119.54 & 1167 & \\
\hline
\end{tabular}

Table 4-3 design speed vs. sensitivity test table

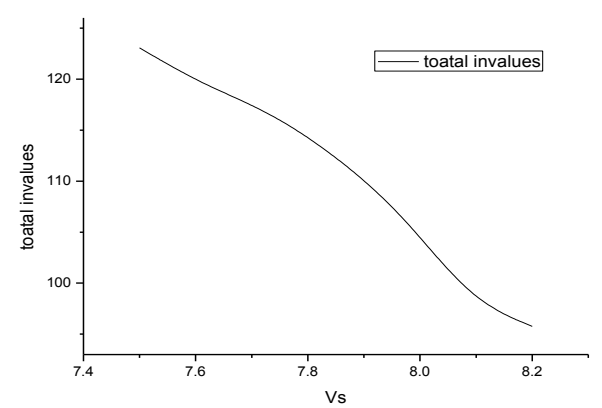

Figure 4-2 trend diagram of the total objective function value B

By analyzing the trend chart, it can be seen that when the variable design speed Vs value is within the range [7.5-8.2], the value of the total objective function shows a decreasing trend, and the decreasing trend is stable. Therefore, when the design speed Vs value is within the range [7.5-8.2], controlling its value around 7.5 is most conducive to the optimization of the value of the total objective function. Controlling other variable intervals unchanged, the propeller diameter Dp is divided into the following seven intervals for optimization. The intervals and corresponding function values c are shown in Table 4-4, and the trend of variation is shown in Figure 4-3 below.

\begin{tabular}{cccccccc}
\hline $\mathrm{D}$ & 0.840 & 0.842 & 0.844 & 0.845 & 0.847 & 0.849 & 0.850 \\
$\mathrm{p}$ & & & & & & & \\
$\mathrm{c}$ & 110.4 & 112.4 & 114.1 & 113.9 & 100.2 & 112.6 & 110.3 \\
& 6 & 3 & 2 & 6 & 8 & 3 & 0 \\
\hline \multicolumn{7}{c}{ Table 4-4 sensitivity test table of propeller diameter Dp }
\end{tabular}

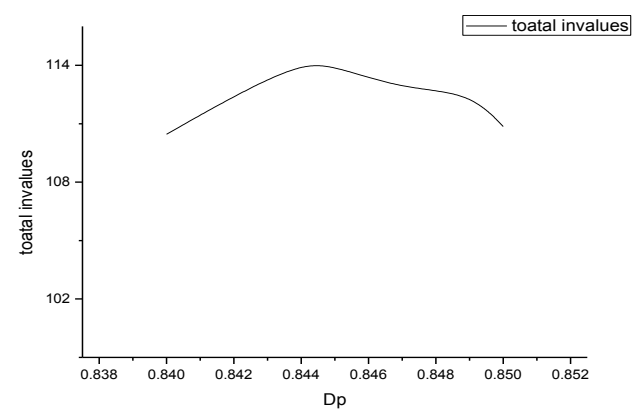

Figure 4-3 change trend diagram of the total objective function value $\mathrm{c}$ 
It can be seen from the analysis of the trend chart that when the variable propeller diameter value is within the interval [0.840-0.850], the total objective function value within the interval [0.84-0.844] presents an increasing trend and the increasing trend is stable. In the interval [0.844-0.850], the value of the total objective function generally shows a decreasing trend, but the decreasing trend is unstable. Therefore, when the propeller diameter value is within the range of [0.840-0.850], the control value around 0.844 is most conducive to the optimization of the total objective function value.

The results of each step are as follows:

Sensitivity of Loa:

$\Delta \mathrm{L}=(\operatorname{amax}-\mathrm{amin}) / \mathrm{amin} * 100 \%=12.12 \%$

Sensitivity of design speed:

$$
\Delta \mathrm{Vs}=(\text { bmax-bmia }) / \text { bmax } * 100 \%=16.53 \%
$$

Sensitivity of propeller diameter:

$$
\Delta \mathrm{Dp}=(\mathrm{cmax}-\mathrm{cmin}) / \mathrm{cmax} * 100 \%=2.56 \%
$$

\section{CONCLUSION}

Sensitivity analysis was carried out on the three variables that had a great impact on the catamaran with small waterplane, namely, Loa, designed speed and propeller diameter. The following conclusions are drawn:

$\mathrm{a} \cdot$ In the comprehensive optimization of small waterplane catamaran with genetic algorithm, the sensitivity of Loa L, design speed Vs and propeller diameter Dp are $12.12 \%, 16.53 \%$ and $2.56 \%$, respectively.

b - Among the three variables of Loa L, design speed Vs and propeller diameter $\mathrm{Dp}$, the one that has the greatest influence on the objective function is design speed Vs.

\section{REFERENCES}

[1] Ma weijia, sun huawei, pang yongjie, guo zhiqun, Yang heng. Optimization method of unmanned boat type [J]. Journal of transportation engineering, 2013,13(1):42-46.

[2] Yang zhuoyi, pang yongjie, wang jian, song lei. Application of response surface model in multi-objective optimization of boat type [J]. Journal of Harbin engineering university. 2011, 32 (4) : 407-438.

[3] Chen kaifeng. Research on improved genetic algorithm and its application in multi-objective optimization [D]. Anhui university.2006.

[4] Goldberg. D. e. netic Algorithms in Search. Optimization and Machine 1_earning. Addison Wesley, 1989

[5] Zhou y h, lu y c, shi z y. adaptive parallel genetic algorithm based on overcoming premature convergence $[\mathrm{J}]$. Journal of tsinghua university (natural science edition),1998,28(3):93-95. (in Chinese with English abstract)

[6] wang xiaogang, liang shixian, wang fuli. Non-inferior hierarchical multi-objective genetic algorithm with local search $[\mathrm{J}]$ northeast university journal (natural science edition),2007,28(7):921-924

[7] CAMPANA E, DIEZ M, LIUZZI G, et al. A multi-objective DIRECT algorithm.

[8] Wu q z. operational research and optimization methods [M]. Beijing: machinery industry press, 2003. (in Chinese)

[9] Cao daoyou. Application research based on improved genetic algorithm [D]. Anhui university,2010. 